\title{
Dynamics of a Stochastic SIS Epidemic Model with Saturated Incidence
}

\author{
Can Chen and Yanmei Kang \\ Department of Applied Mathematics, School of Mathematics and Statistics, Xian Jiaotong University, Xian 710049, China
}

Correspondence should be addressed to Yanmei Kang; ymkang@mail.xjtu.edu.cn

Received 6 April 2014; Accepted 19 May 2014; Published 15 June 2014

Academic Editor: Debora Amadori

Copyright (c) 2014 C. Chen and Y. Kang. This is an open access article distributed under the Creative Commons Attribution License, which permits unrestricted use, distribution, and reproduction in any medium, provided the original work is properly cited.

\begin{abstract}
We introduce stochasticity into the SIS model with saturated incidence. The existence and uniqueness of the positive solution are proved by employing the Lyapunov analysis method. Then, we carry out a detailed analysis on both its almost sure exponential stability and its $p$ th moment exponential stability, which indicates that the $p$ th moment exponential stability implies the almost sure exponential stability. Additionally, the results show that the conditions for the disease to become extinct are much weaker than those in the corresponding deterministic model. The conditions for the persistence in the mean and the existence of a stationary distribution are also established. Finally, we derive the expressions for the mean and variance of the stationary distribution. Compared with the corresponding deterministic model, the threshold value for the disease to die out is affected by the half saturation constant. That is, increasing the saturation effect can reduce the disease transmission. Computer simulations are presented to illustrate our theoretical results.
\end{abstract}

\section{Introduction}

Epidemiology has been investigated by mathematicians through establishing mathematical models for a long time; see, for instance, [1-4]. Mathematical models can help people to better understand the spread of the disease and thus take effective measures to reduce its transmission as much as possible. Particularly, the classical SIS epidemic model $[5,6]$ is often used to model the dynamics of the diseases with no protective immunity such as gonorrhea.

Usually, the incidence function describes the number of new infections per unit time and it plays a vital role in the deterministic models. As it is known, in the most existing literature, the bilinear incidence rate $\beta S I$ is frequently used. However, this kind of incidence rate has some limitations, since it does not consider the behavioral change of susceptible individuals or control measures taken by the government when the number of infective individuals gets large. For example, people would wash their hands frequently, wear masks, and limit their time of going out, and the government would also take measures such as quarantine and isolation when the A/H1N1 influenza became serious in 2009 [7]. Therefore, it is more reasonable to adopt saturated incidence rate $\beta S I /(1+h I)[8]$ in the mathematical models. When the number of infective individuals gets large, $\beta I /(1+h I)$ tends to a saturation effect, where $\beta I$ is the infection force of the disease and $1 /(1+h I)$ is the inhibition effect from the behavioral change or control measures. Here, $h$ represents the half saturation constant [9]. With the saturated incidence taken into consideration, the classical SIS epidemic model is transformed into

$$
\begin{gathered}
\frac{d S(t)}{d t}=\mu N-\frac{\beta S(t) I(t)}{1+h I(t)}+\gamma I(t)-\mu S(t), \\
\frac{d I(t)}{d t}=\frac{\beta S(t) I(t)}{1+h I(t)}-(\mu+\gamma) I(t),
\end{gathered}
$$

where $S(t)$ and $I(t)$ are the number of susceptible individuals and infective individuals at time $t$, respectively. The total population $N=S(t)+I(t)$ is a constant. Consider the following parameters: $\beta$ is the transmission rate, $\mu$ is the birth and death rate, and $\gamma$ is the recovery rate. Assume all these parameters are positive; then, the classical stability 
analysis has shown that the system (1) always has a disease free equilibrium:

$$
E_{0}=\left(S_{0}, I_{0}\right)=(N, 0)
$$

The basic reproduction number, $R_{0}^{d}=\beta N /(\mu+\gamma)$, is a threshold value, which determines the extinction and persistence of the disease. When $R_{0}^{d} \leq 1$, the disease free equilibrium $E_{0}$ is globally stable. When $R_{0}^{d}>1$, the disease free equilibrium $E_{0}$ is unstable, and model (1) has a unique endemic equilibrium:

$$
E^{*}=\left(S^{*}, I^{*}\right)=\left(\frac{(1+h N)(\mu+\gamma)}{\beta+h(\mu+\gamma)}, \frac{\beta N-(\mu+\gamma)}{\beta+h(\mu+\gamma)}\right),
$$

which is globally stable.

Since the noise exists almost everywhere, epidemic models are inevitably affected by it. Hence, it is more reasonable to introduce random perturbations into mathematical models. At the same time, we note that taking the effect of environment noise on epidemic models into consideration has been a popular trend in disease spread modeling [10-13]. Gray et al. presented a stochastic SIS epidemic model in [14]. They established conditions for extinction and persistence of the disease and the existence of a stationary distribution. Stochastic SIR and SIRS models with and without distributed time delay were investigated in $[15,16]$, respectively, where the asymptotic behavior was discussed. In [17], stochastically perturbed SIR and SEIR epidemic models with saturated incidence were studied and the results of extinction and ergodicity were concluded. Ji et al. investigated a stochastic multigroup SIR model with fluctuations around the transmission coefficient and the death rate of each subpopulation in $[18,19]$ separately. Zhao et al. [20] discussed the extinction and persistence of the stochastic SIS epidemic model with vaccination. However, among all these researches, there are few literatures considering the parameter perturbation in SIS epidemic model with saturated incidence.

Motivated by the above consideration, in this paper, we introduce random effect into the SIS model (1) by replacing the transmission rate $\beta$ with $\beta+\sigma d W(t)$, where $W(t)$ is standard Brownian motion and $\sigma$ is the intensity of white noise. The stochastic version corresponding to the deterministic model (1) can be represented as

$$
\begin{aligned}
d S(t)= & \left(\mu N-\frac{\beta S(t) I(t)}{1+h I(t)}+\gamma I(t)-\mu S(t)\right) d t \\
& -\frac{\sigma S(t) I(t)}{1+h I(t)} d W(t), \\
d I(t)= & \left(\frac{\beta S(t) I(t)}{1+h I(t)}-(\mu+\gamma) I(t)\right) d t+\frac{\sigma S(t) I(t)}{1+h I(t)} d W(t) .
\end{aligned}
$$

Given that $S(t)+I(t)=N$, it is sufficient to study the following equation:

$$
\begin{aligned}
d I(t)= & \left(\frac{\beta(N-I(t)) I(t)}{1+h I(t)}-(\mu+\gamma) I(t)\right) d t \\
& +\frac{\sigma(N-I(t)) I(t)}{1+h I(t)} d W(t),
\end{aligned}
$$

with initial value $I(0)=I_{0} \in(0, N)$. In the following sections, we will restrict ourselves to (5).

The paper is organized as follows. In Section 2, the existence of unique positive solution is shown. Both its almost sure exponential stability and its $p$ th moment exponential stability are then investigated in Section 3. The conditions for persistence in the mean of the disease are established in Section 4. The existence of a stationary distribution and the expressions for the mean and variance are presented in Section 5. In Section 6, we give a brief conclusion. Besides, the computer simulations which support our results are given in each section. Finally, we give an appendix containing some theory used in the previous sections.

\section{Existence of Unique Positive Solution}

To investigate the dynamical behavior of the epidemic model, we need to show that the model has a unique global solution and the solution will remain within $(0, N)$ whenever it starts there. Hence, in this section, employing the Lyapunov analysis method (mentioned in $[18,21]$ ), we establish Theorem 1 .

Theorem 1. There is a unique global solution $I(t) \in(0, N)$ of system (5) on $t \geq 0$ for any given initial value $I(0)=I_{0} \in$ $(0, N)$ with probability 1; namely,

$$
P\{I(t) \in(0, N), \forall t \geq 0\}=1 .
$$

Proof. Since the coefficients of (5) are locally Lipschitz continuous for any given initial value $I(0)=I_{0} \in(0, N)$, there is a unique local solution $I(t)$ on $t \in\left[0, \tau_{e}\right)$, where $\tau_{e}$ is the explosion time [22]. To show that the solution is global, we need to show that $\tau_{e}=\infty$ a.s. Let $m_{0}>0$ be sufficiently large such that $1 / m_{0}<I_{0}<N-1 / m_{0}$. For each integer $m \geq m_{0}$, define the stopping time

$$
\tau_{m}=\inf \left\{t \in\left[0, \tau_{e}\right): I(t) \notin\left(\frac{1}{m}, N-\frac{1}{m}\right)\right\},
$$

where throughout this paper we set inf $\emptyset=\infty$ (as usual $\emptyset$ denotes the empty set). Clearly, $\tau_{m}$ is increasing as $m \rightarrow \infty$. Set $\tau_{\infty}=\lim _{m \rightarrow \infty} \tau_{m}$, whence $\tau_{\infty} \leq \tau_{e}$ a.s. If we can show that $\tau_{\infty}=\infty$ a.s., then $\tau_{e}=\infty$ a.s. and $I(t) \in(0, N)$ a.s. for all $t \geq 0$. In other words, to complete the proof, all what we need to show is that $\tau_{\infty}=\infty$ a.s. If this statement is not true, then there exists a pair of constants $T>0$ and $\epsilon \in(0,1)$ such that

$$
P\left\{\tau_{\infty} \leq T\right\}>\epsilon .
$$

Therefore, there is an integer $m_{1} \geq m_{0}$ such that

$$
P\left\{\tau_{m} \leq T\right\} \geq \epsilon, \quad \forall m \geq m_{1} .
$$


Define a function $V:(0, N) \rightarrow R_{+}$by

$$
V(x)=\frac{1}{x}+\frac{1}{N-x} \text {. }
$$

Using the Itô formula, we get

$$
\begin{aligned}
d V(x)= & \left(-\frac{1}{x^{2}}+\frac{1}{(N-x)^{2}}\right) d x+\left(\frac{1}{x^{3}}+\frac{1}{(N-x)^{3}}\right)(d x)^{2} \\
= & L V(x) d t \\
& +\left(-\frac{\sigma(N-x)}{x(1+h x)}+\frac{\sigma x}{(N-x)(1+h x)}\right) d W(t),
\end{aligned}
$$

where $L V:(0, N) \rightarrow R$ is defined by

$$
\begin{aligned}
L V= & \left(-\frac{1}{x^{2}}+\frac{1}{(N-x)^{2}}\right)\left(\frac{\beta(N-x) x}{1+h x}-(\mu+\gamma) x\right) \\
& +\left(\frac{1}{x^{3}}+\frac{1}{(N-x)^{3}}\right) \frac{\sigma^{2}(N-x)^{2} x^{2}}{(1+h x)^{2}} \\
= & -\frac{\beta(N-x)}{x(1+h x)}+\frac{\mu+\gamma}{x}+\frac{\beta x}{(N-x)(1+h x)} \\
& +\frac{\sigma^{2}(N-x)^{2}}{x(1+h x)^{2}}+\frac{\sigma^{2} x^{2}}{(N-x)(1+h x)^{2}}-\frac{(\mu+\gamma) x}{(N-x)^{2}} \\
\leq & \frac{\mu+\gamma}{x}+\frac{\beta x}{(N-x)(1+h x)}+\frac{\sigma^{2}(N-x)^{2}}{x(1+h x)^{2}} \\
& +\frac{\sigma^{2} x^{2}}{(N-x)(1+h x)^{2}} \\
\leq & \frac{1}{x}\left(\mu+\gamma+\sigma^{2} N^{2}\right)+\frac{1}{N-x}\left(\frac{\beta}{h}+\frac{\sigma^{2}}{h^{2}}\right) \\
\leq & C V(x),
\end{aligned}
$$

where $C=\left(\mu+\gamma+\sigma^{2} N^{2}\right) \vee\left(\beta / h+\sigma^{2} / h^{2}\right)$. Therefore,

$$
\begin{array}{rl}
\int_{0}^{\tau_{m} \wedge t} & d V(I(s)) \\
= & \int_{0}^{\tau_{m} \wedge t} L V(I(s)) d s \\
& +\int_{0}^{\tau_{m} \wedge t}\left(-\frac{\sigma(N-I(s))}{I(s)(1+h I(s))}\right) d W(s) \\
\leq & \left.+\frac{\sigma I(s)}{(N-I(s))(1+h I(s))}\right) \\
& +\int_{0}^{\tau_{m} \wedge t} C V(I(s)) d s \\
& +\frac{\sigma(N-I(s))}{I(s)(1+h I(s))} \\
& \left.+\frac{\sigma I(s)}{(N-I(s))(1+h I(s))}\right) d W(s),
\end{array}
$$

which implies that

$$
\begin{aligned}
E V\left(I\left(\tau_{m} \wedge t\right)\right) & \leq V\left(I_{0}\right)+C E \int_{0}^{\tau_{m} \wedge t} V(I(s)) d s \\
& \leq V\left(I_{0}\right)+C \int_{0}^{t} E V\left(I\left(\tau_{m} \wedge s\right)\right) d s
\end{aligned}
$$

The Gronwall inequality yields that

$$
E V\left(I\left(\tau_{m} \wedge T\right)\right) \leq V\left(I_{0}\right) e^{C T} .
$$

Set $\Omega_{m}=\left\{\tau_{m} \leq T\right\}$ for all $m \geq m_{1}$. Then, by (9), $P\left(\Omega_{m}\right) \geq \epsilon$. For every $\omega \in \Omega_{m}, I\left(\tau_{m}, \omega\right)$ equals either $1 / m$ or $N-1 / m$, and therefore

$$
V\left(I\left(\tau_{m}, \omega\right)\right) \geq m
$$

It then follows from (9) and (15) that

$$
V\left(I_{0}\right) e^{C T} \geq E\left[1_{\Omega_{m}(\omega)} V\left(I\left(\tau_{m}, \omega\right)\right)\right] \geq \epsilon m,
$$

where $1_{\Omega_{m}(\omega)}$ is the indicator function of $\Omega_{m}$. Letting $m \rightarrow$ $\infty$ leads to the contradiction that

$$
\infty>V\left(I_{0}\right) e^{C T}=\infty
$$

So, we must have $\tau_{\infty}=\infty$ a.s., whence the proof is complete.

\section{Extinction}

In discussing the extinction of system (5), we focus on the two kinds of exponential stabilities, almost sure exponential stability and $p$ th moment exponential stability.

\subsection{Almost Sure Exponential Stability}

Theorem 2. Let $I(t)$ be the solution of system (5) with initial value $I(0)=I_{0} \in(0, N)$. If

(a) $R_{01}^{s}:=\beta N /(\mu+\gamma)-\sigma^{2} N^{2} /\left(2(\mu+\gamma)(1+h N)^{2}\right)=$ $R_{0}^{d}-\sigma^{2} N^{2} /\left(2(\mu+\gamma)(1+h N)^{2}\right)<1$ and $\sigma^{2} \leq$ $\beta(1+h N)^{2} / N$,

(b) $\sigma^{2}>\beta(1+h N)^{2} / N \vee \beta^{2}(1+h N)^{2} / 2(\mu+\gamma)$.

then,

$$
\begin{aligned}
& \lim \sup _{t \rightarrow \infty}(\log (I(t)) / t) \leq \beta N-\mu-\gamma-\sigma^{2} N^{2} / \\
& 2(1+h N)^{2}<0 \quad \text { a.s. if }(a) \text { holds; } \\
& \limsup _{t \rightarrow \infty}(\log (I(t)) / t) \leq \beta^{2}(1+h N)^{2} / 2 \sigma^{2}-\mu-\gamma< \\
& 0 \text { a.s. if (b) holds; }
\end{aligned}
$$

that is, $I(t)$ tends to zero exponentially a.s.; namely, the disease will die out with probability one.

Proof. Applying Itô formula to system (5) leads to

$$
d \log (I(t))=F(I(t)) d t+\frac{\sigma(N-I(t))}{1+h I(t)} d W(t),
$$


where $F(I(t))=\beta(N-I(t)) /(1+h I(t))-\mu-\gamma-\sigma^{2}$ $(N-I(t))^{2} / 2(1+h I(t))^{2}$. Integrating both sides of (19) from 0 to $t$, we have

$$
\log (I(t))=\log \left(I_{0}\right)+\int_{0}^{t} F(I(s)) d s+M(t),
$$

where $M(t)=\int_{0}^{t}(\sigma(N-I(s)) /(1+h I(s))) d W(s)$. Obviously, $F(I(s)) \leq \beta N-\beta I(s)-\mu-\gamma-\frac{\sigma^{2}(N-I(s))^{2}}{2(1+h N)^{2}}:=G(I(s))$,

where $G(I(s))=\beta N-\mu-\gamma-\sigma^{2} N^{2} / 2(1+h N)^{2}-(\beta-$ $\left.\sigma^{2} N /(1+h N)^{2}\right) I(s)-\left(\sigma^{2} / 2(1+h N)^{2}\right) I(s)^{2}$, for $I(s) \in(0, N)$.

If condition (a) is satisfied, it then follows from (20) that

$$
\begin{aligned}
\log (I(t)) \leq & \log \left(I_{0}\right) \\
& +\left(\beta N-\mu-\gamma-\frac{\sigma^{2} N^{2}}{2(1+h N)^{2}}\right) t+M(t) .
\end{aligned}
$$

This implies that

$$
\begin{aligned}
\limsup _{t \rightarrow \infty} \frac{\log (I(t))}{t} \leq & \beta N-\mu-\gamma-\frac{\sigma^{2} N^{2}}{2(1+h N)^{2}} \\
& +\limsup _{t \rightarrow \infty} \frac{M(t)}{t} \text { a.s. }
\end{aligned}
$$

Clearly, $M(t)=\int_{0}^{t}(\sigma(N-I(s)) /(1+h I(s))) d W(s)$ is a local martingale and vanishes at $t=0$. Moreover,

$$
\limsup _{t \rightarrow \infty} \frac{\langle M, M\rangle_{t}}{t} \leq \sigma^{2} N^{2}<\infty \quad \text { a.s. }
$$

By strong law of large numbers [22], we get

$$
\lim _{t \rightarrow \infty} \frac{M(t)}{t}=0 \quad \text { a.s. }
$$

Then,

$$
\limsup _{t \rightarrow \infty} \frac{\log (I(t))}{t} \leq \beta N-\mu-\gamma-\frac{\sigma^{2} N^{2}}{2(1+h N)^{2}}<0 \quad \text { a.s. }
$$

If condition (b) is satisfied, the function $G(x)$ takes its maximum value $G(\tilde{x})$ at $\left.\tilde{x}=N-\beta(1+h N)^{2} / \sigma^{2} \in(0, N)\right)$; that is, $G(\tilde{x})=\beta^{2}(1+h N)^{2} / 2 \sigma^{2}-(\mu+\gamma)$. It then follows from (20) that

$$
\log (I(t)) \leq \log \left(I_{0}\right)+\left(\frac{\beta^{2}(1+h N)^{2}}{2 \sigma^{2}}-(\mu+\gamma)\right) t+M(t)
$$

Then,

$$
\limsup _{t \rightarrow \infty} \frac{\log (I(t))}{t} \leq \frac{\beta^{2}(1+h N)^{2}}{2 \sigma^{2}}-\mu-\gamma<0 \text { a.s. }
$$

This finishes the proof.
From Theorem 2, we know that when $R_{01}^{s}=R_{0}^{d}-\sigma^{2} N^{2} /$ $2(\mu+\gamma)(1+h N)^{2}<1$ and the perturbation is small, the disease will die out a.s., whereas the disease may still persist for the corresponding deterministic model (1). If the noise intensity $\sigma^{2}$ is larger than $\beta(1+h N)^{2} / N \vee \beta^{2}(1+h N)^{2} / 2(\mu+$ $\gamma)$, then the disease will also die out a.s. For $h=0$, system (5) becomes the model discussed in [14] and the conditions for the disease to become extinct we obtain here are in agreement with those derived in [14]. If there exists no environment noise, that is, $\sigma=0$, then $R_{01}^{s}=R_{0}^{d}$ is the basic reproduction number of the deterministic model (1). In addition, the half saturation constant also influences $R_{01}^{s}$, which shows that increasing the saturation effect can reduce the value of $R_{01}^{s}$ and thus can reduce the disease spread. The following simulations indicate these results.

Example 3. We assume that the unit time is one day. The parameters are given by

$$
\begin{aligned}
& N=100, \quad \beta=0.013, \quad \mu=0.4, \\
& \gamma=0.6, \quad h=0.05, \quad \sigma=0.06 \text {. }
\end{aligned}
$$

Note that

$$
\begin{gathered}
R_{01}^{s}=\frac{\beta N}{\mu+\gamma}-\frac{\sigma^{2} N^{2}}{2(\mu+\gamma)(1+h N)^{2}}=0.8<1, \\
\sigma^{2}=0.0036 \leq \frac{\beta(1+h N)^{2}}{N}=0.0047 .
\end{gathered}
$$

That is, condition (a) is satisfied and the solution $I(t)$ satisfies

$$
\begin{aligned}
\limsup _{t \rightarrow \infty} \frac{\log (I(t))}{t} & \leq \beta N-\mu-\gamma-\frac{\sigma^{2} N^{2}}{2(1+h N)^{2}} \\
& =-0.2<0 \quad \text { a.s. }
\end{aligned}
$$

Hence, $I(t)$ tends to zero exponentially a.s.; that is, the disease will die out a.s.

On the other hand, for the deterministic model (1), the basic reproduction number is

$$
R_{0}^{d}=\frac{\beta N}{\mu+\gamma}=1.3>1 .
$$

The solution $I(t)$ obeys

$$
\lim _{t \rightarrow \infty} I(t)=\frac{\beta N-(\mu+\gamma)}{\beta+(\mu+\gamma) h}=4.7619 .
$$

Employing the Euler-Maruyama (EM) method in [23], the computer simulations are given in Figure 1, which support our results.

Example 4. We keep the parameters the same as Example 3 but increase $\sigma$ to 0.09 . Note that

$\sigma^{2}=0.0081$

$$
\begin{aligned}
& >\max \left\{\frac{\beta(1+h N)^{2}}{N}=0.00468, \frac{\beta^{2}(1+h N)^{2}}{2(\mu+\gamma)}=0.00304\right\} \\
& =0.00468 .
\end{aligned}
$$




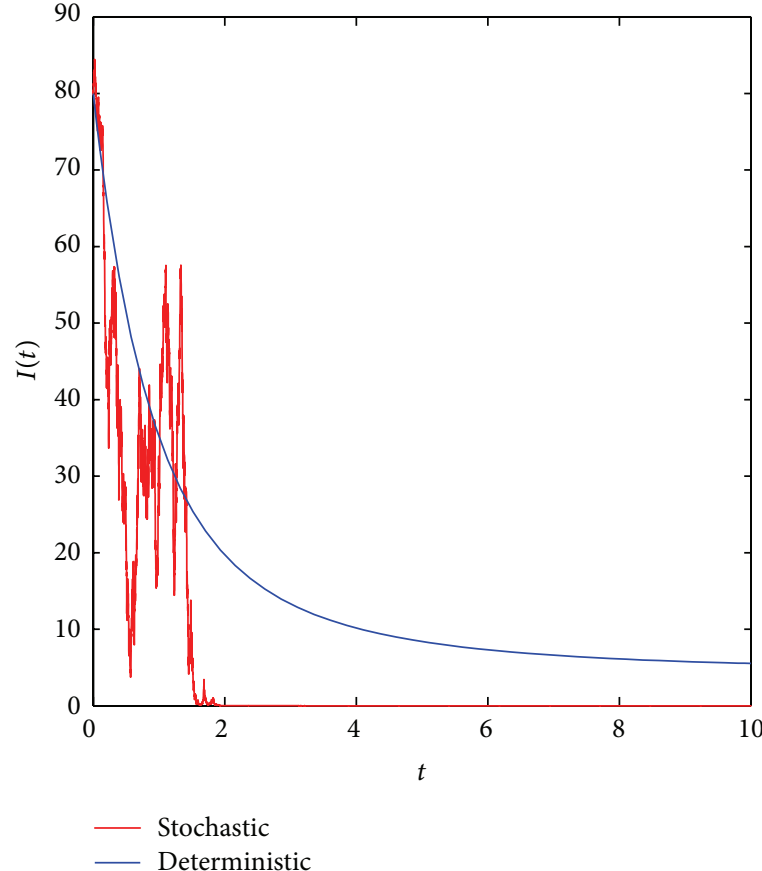

(a)

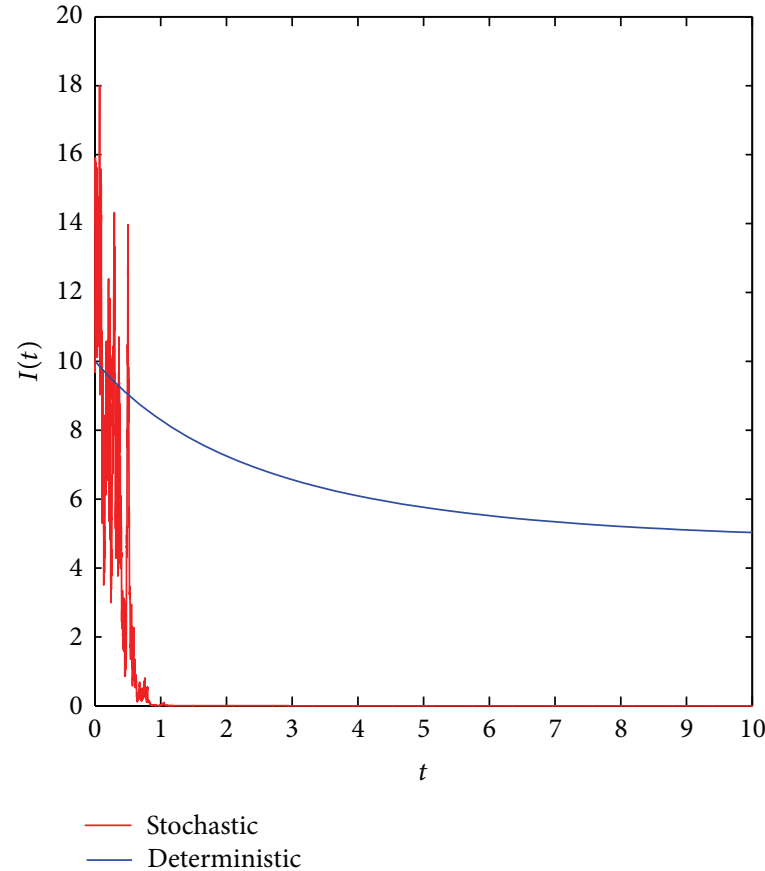

(b)

Figure 1: Computer simulations of $I(t)$ for model (5) and the deterministic model (1), using the EM method with step size 0.001 and with initial values (a) $I(0)=80$ and (b) $I(0)=10$.

That is, condition (b) is satisfied and

$$
\begin{aligned}
\limsup _{t \rightarrow \infty} & \frac{\log (I(t))}{t} \\
\leq & \frac{\beta^{2}(1+h N)^{2}}{2 \sigma^{2}}-\mu-\gamma=-0.624<0 \quad \text { a.s. }
\end{aligned}
$$

Therefore, $I(t)$ tends to zero exponentially a.s.; namely, the disease will die out a.s. The simulations are shown in Figure 2 to support our results completely.

3.2. pth Moment Exponential Stability. Following (20), we obtain

$$
I(t)=I_{0} \exp \left(\int_{0}^{t} F(I(s)) d s+M(t)\right)
$$

As $W(t) \sim N(0, t)$, then $[24]$

$$
\begin{aligned}
E e^{M(t)} & =E \exp \left(\int_{0}^{t} \frac{\sigma(N-I(s))}{1+h I(s)} d W(s)\right) \\
& \leq E e^{\sigma N W(t)}=e^{\left(\sigma^{2} N^{2} / 2\right) t} .
\end{aligned}
$$

Using the fact that

$$
F(I(s)) \leq \beta N-\mu-\gamma,
$$

we find

$$
\begin{aligned}
E I(t) & =E I_{0} \exp \left(\int_{0}^{t} F(I(s)) d s+M(t)\right) \\
& \leq I_{0} \exp \left(\left(\beta N-\mu-\gamma+\frac{\sigma^{2} N^{2}}{2}\right) t\right) .
\end{aligned}
$$

Since

$$
\begin{aligned}
\log (I(t))^{p} & =p \log (I(t)) \\
& \leq p\left(\log \left(I_{0}\right)+(\beta N-\mu-\gamma) t+\sigma N W(t)\right)
\end{aligned}
$$

then

$$
\begin{aligned}
E(I(t))^{p} & \leq\left(I_{0}\right)^{p} \cdot e^{p(\beta N-\mu-\gamma) t} \cdot e^{\left(p^{2} \sigma^{2} N^{2} / 2\right) t} \\
& =\left(I_{0}\right)^{p} \exp \left(p\left(\beta N-\mu-\gamma+\frac{p \sigma^{2} N^{2}}{2}\right) t\right) .
\end{aligned}
$$

Theorem 5. Assume that $\beta N-\mu-\gamma+\left(p \sigma^{2} N^{2} / 2\right)<0$; the solution $I(t)$ with initial value $I(0)=I_{0} \in(0, N)$ is pth moment exponentially stable. 


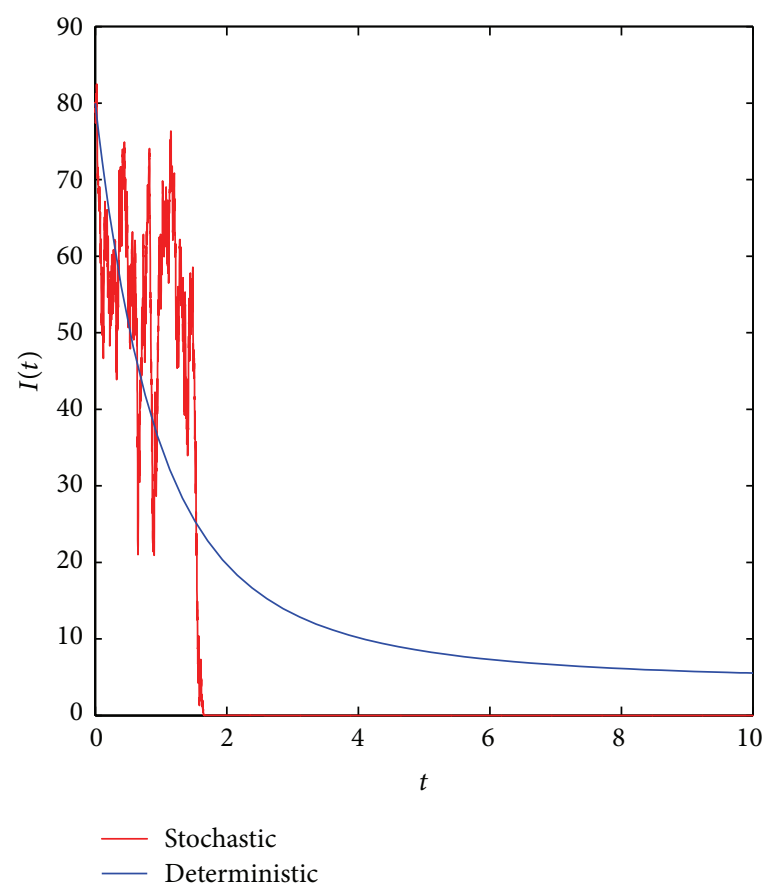

(a)

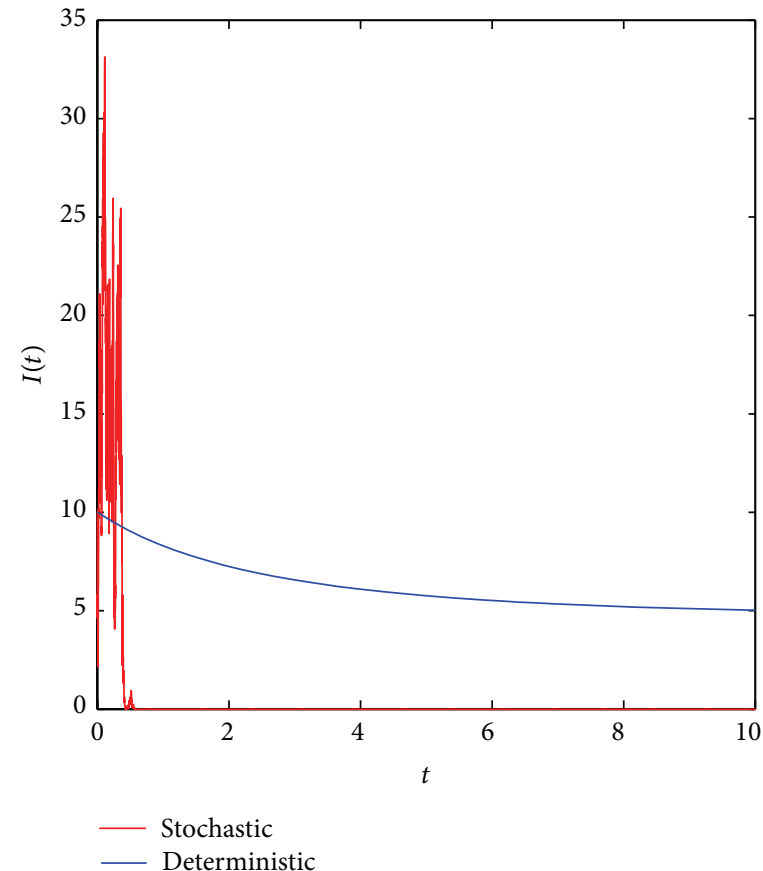

(b)

Figure 2: Computer simulations of $I(t)$ for model (5) and the deterministic model (1), using the EM method with step size 0.001 and with initial values (a) $I(0)=80$ and (b) $I(0)=10$.

Next, we will make a comparison between the almost sure exponential stability and the $p$ th moment exponential stability, since

$$
\begin{aligned}
x\left(\frac{\beta(N-x) x}{1+h x}-(\mu+\gamma) x\right) & =\left(\frac{\beta(N-x)}{1+h x}-(\mu+\gamma)\right) x^{2} \\
& \leq(\beta N-(\mu+\gamma)) x^{2} .
\end{aligned}
$$

From Theorem 5, we know that $\beta N-(\mu+\gamma)<0$; that is,

$$
x\left(\frac{\beta(N-x) x}{1+h x}-(\mu+\gamma) x\right) \leq(\beta N-(\mu+\gamma)) x^{2} \leq 0 .
$$

Moreover,

$$
\frac{\sigma^{2}(N-x)^{2} x^{2}}{(1+h x)^{2}} \leq \sigma^{2}(N-x)^{2} x^{2} \leq \sigma^{2} N^{2} x^{2} .
$$

Let $K=\sigma^{2} N^{2}$ be positive; then

$$
x\left(\frac{\beta(N-x) x}{1+h x}-(\mu+\gamma) x\right) \vee \frac{\sigma^{2}(N-x)^{2} x^{2}}{(1+h x)^{2}} \leq K x^{2} .
$$

Therefore, the condition of Lemma A.4 (in the appendix) [22] is satisfied, which suggests that the $p$ th moment exponential stability of system (5) implies the almost sure exponential stability.

\section{Persistence}

We will investigate persistence in the mean for system (5) in this section. The definition of the persistence in the mean was initially proposed for the deterministic system [25] and was also used for the stationary stochastic system with ergodicity $[19,20]$. According to the ergodicity for the stationary process, the time average in the long time limit is equal to the ensemble expectation over phase space, so we introduce the notation

$$
\langle I(t)\rangle=\frac{1}{t} \int_{0}^{t} I(s) d s .
$$

Definition 6. System (5) is said to be persistent in the mean, if

$$
\liminf _{t \rightarrow \infty} \frac{1}{t} \int_{0}^{t} I(s) d s>0 \quad \text { a.s. }
$$

Theorem 7. If

$$
\begin{gathered}
R_{02}^{s}:=\frac{\beta N}{(\mu+\gamma)(1+h N)}-\frac{\sigma^{2} N^{2}}{2(\mu+\gamma)}>1, \\
\sigma^{2}<\frac{\beta(1+h N)^{2}}{N},
\end{gathered}
$$

then, for any initial value $I(0)=I_{0} \in(0, N)$, the solution $I(t)$ of system (5) has the following property:

$$
\underline{I} \leq \liminf _{t \rightarrow \infty}\langle I(t)\rangle \leq \limsup _{t \rightarrow \infty}\langle I(t)\rangle \leq \bar{I} \quad \text { a.s. }
$$


where

$$
\begin{aligned}
& \bar{I}=\frac{\beta N-\mu-\gamma-\left(\sigma^{2} N^{2} / 2(1+h N)^{2}\right)}{\beta-\left(\sigma^{2} N /(1+h N)^{2}\right)}, \\
& \underline{I}=\frac{\beta N /(1+h N)-\mu-\gamma-\left(\sigma^{2} N^{2} / 2\right)}{\beta /(1+h N)} .
\end{aligned}
$$

Proof. By (20), we obtain

$$
\begin{aligned}
\frac{\log (I(t))-\log \left(I_{0}\right)}{t} \\
\leq \frac{1}{t} \int_{0}^{t}\left(\beta N-\beta I(s)-\mu-\gamma-\frac{\sigma^{2}(N-I(s))^{2}}{2(1+h N)^{2}}\right) d s \\
\quad+\frac{M(t)}{t} \\
=\beta N-\mu-\gamma-\frac{\sigma^{2} N^{2}}{2(1+h N)^{2}}-\beta\langle I(t)\rangle \\
\quad+\frac{\sigma^{2} N}{(1+h N)^{2}}\langle I(t)\rangle-\frac{\sigma^{2}}{2(1+h N)^{2}}\left\langle I(t)^{2}\right\rangle+\frac{M(t)}{t} \\
\leq \beta N-\mu-\gamma-\frac{\sigma^{2} N^{2}}{2(1+h N)^{2}}-\left(\beta-\frac{\sigma^{2} N}{(1+h N)^{2}}\right)\langle I(t)\rangle \\
\quad+\frac{M(t)}{t} .
\end{aligned}
$$

The inequality can be written as

$$
\begin{aligned}
\frac{\log (I(t))}{t} \leq & \beta N-\mu-\gamma-\frac{\sigma^{2} N^{2}}{2(1+h N)^{2}} \\
& -\left(\beta-\frac{\sigma^{2} N}{(1+h N)^{2}}\right)\langle I(t)\rangle+\frac{M(t)}{t}+\frac{\log \left(I_{0}\right)}{t} .
\end{aligned}
$$

Since $R_{01}^{s} \geq R_{02}^{s}$, if condition (48) is satisfied, then

$$
\beta N-\mu-\gamma-\frac{\sigma^{2} N^{2}}{2(1+h N)^{2}}>0, \quad \beta-\frac{\sigma^{2} N}{(1+h N)^{2}}>0 .
$$

Together with Lemma A.2. in [20], we have

$\lim _{t \rightarrow \infty} \sup \langle I(t)\rangle$

$$
\leq \frac{\beta N-\mu-\gamma-\left(\sigma^{2} N^{2} / 2(1+h N)^{2}\right)}{\beta-\left(\sigma^{2} N /(1+h N)^{2}\right)}=\bar{I} \quad \text { a.s. }
$$

On the other hand,

$$
\begin{aligned}
& \frac{\log (I(t))-\log \left(I_{0}\right)}{t} \\
& \geq \frac{1}{t} \int_{0}^{t}\left(\frac{\beta(N-I(s))}{1+h N}-\mu-\gamma-\frac{1}{2} \sigma^{2}(N-I(s))^{2}\right) d s \\
& \quad+\frac{M(t)}{t} \\
& \geq \frac{\beta N}{1+h N}-\mu-\gamma-\frac{1}{2} \sigma^{2} N^{2}-\frac{\beta}{1+h N}\langle I(t)\rangle+\frac{M(t)}{t} .
\end{aligned}
$$

That is,

$$
\begin{aligned}
\langle I(t)\rangle \geq \frac{1}{\beta /(1+h N)}( & \frac{\beta N}{1+h N}-\mu-\gamma-\frac{1}{2} \sigma^{2} N^{2} \\
& \left.+\frac{M(t)}{t}-\frac{\log (I(t))-\log \left(I_{0}\right)}{t}\right) .
\end{aligned}
$$

Since $-\infty<\log (I(t))<\log (N)$, then

$$
\begin{aligned}
\liminf _{t \rightarrow \infty}\langle I(t)\rangle & \geq \frac{\beta N /(1+h N)-\mu-\gamma-\left(\sigma^{2} N^{2} / 2\right)}{\beta /(1+h N)} \\
& =\underline{I} \in(0, N) \quad \text { a.s. }
\end{aligned}
$$

Moreover,

$$
\begin{aligned}
\bar{I} & =\frac{\beta N-\mu-\gamma-\left(\sigma^{2} N^{2} / 2(1+h N)^{2}\right)}{\beta-\left(\sigma^{2} N /(1+h N)^{2}\right)} \\
& \geq \underline{I}=\frac{\beta N /(1+h N)-\mu-\gamma-\left(\sigma^{2} N^{2} / 2\right)}{\beta /(1+h N)} .
\end{aligned}
$$

Therefore, we complete the proof.

Example 8. The parameters are given by

$$
\begin{aligned}
& N=100, \quad \beta=0.2, \quad \mu=0.4 \text {, } \\
& \gamma=0.6, \quad h=0.05, \quad \sigma=0.02 .
\end{aligned}
$$

Note that

$$
\begin{gathered}
R_{02}^{s}:=\frac{\beta N}{(\mu+\gamma)(1+h N)}-\frac{\sigma^{2} N^{2}}{2(\mu+\gamma)}=1.33>1, \\
\sigma^{2}=0.0004<\frac{\beta(1+h N)^{2}}{N}=0.072 .
\end{gathered}
$$

Then the solution $I(t)$ of system (5) satisfies

$$
10 \leq \liminf _{t \rightarrow \infty}\langle I(t)\rangle \leq \limsup _{t \rightarrow \infty}\langle I(t)\rangle \leq 95.2938 \text { a.s. }
$$


For the corresponding deterministic model (1),

$$
\begin{gathered}
R_{0}^{d}=\frac{\beta N}{(\mu+\gamma)}=20>1, \\
\lim _{t \rightarrow \infty} I(t)=\frac{\beta N-(\mu+\gamma)}{\beta+h(\mu+\gamma)}=76 .
\end{gathered}
$$

The simulation results are shown in Figure 3.

\section{Stationary Distribution}

Applying Lemma A.5 (in the appendix) [26], if we can show that conditions (B.1) and (B.2) are satisfied, then system (5) has a stationary distribution. Obviously, the square of the diffusion coefficient of system (5), that is, $\sigma^{2}(N-I)^{2} I^{2} /(1+h I)^{2}$, is bounded away from zero for $I \in$ $(a, b) \subset(0, N))$; then, condition (B.1) is satisfied. Next, we will prove that (B.2) is also valid.

Theorem 9. Assume that $R_{02}^{s}:=\beta N /((\mu+\gamma)(1+h N))-$ $\sigma^{2} N^{2} /(2(\mu+\gamma))>1$ and $\sigma^{2}<\left(2 \beta /\left(N^{2}\left(1+h I^{*}\right)\right.\right.$ $\left.\left.I^{*}\right)\right) \min \left\{\left(I^{*}\right)^{2},\left(N-I^{*}\right)^{2}\right\}$; then, there is a unique stationary distribution of system (5). Here, $I^{*}$ is the unique endemic equilibrium of system (1); that is, $I^{*}=(\beta N-(\mu+\gamma)) /(\beta+$ $h(\mu+\gamma))$. Moreover,

$$
\lim _{t \rightarrow \infty} \frac{1}{t} E \int_{0}^{t} \frac{\beta}{1+h I^{*}}\left(I(s)-I^{*}\right)^{2} d s \leq \frac{1}{2} I^{*} \sigma^{2} N^{2}
$$

Proof. If $R_{02}^{s}:=\beta N /((\mu+\gamma)(1+h N))-\sigma^{2} N^{2} / 2(\mu+\gamma)>1$, it is clear that $R_{0}^{d}=\beta N /(\mu+\gamma)>1$; then, model (1) has a unique endemic equilibrium $I^{*}$; that is,

$$
\frac{\beta\left(N-I^{*}\right)}{1+h I^{*}}=\mu+\gamma, \quad I^{*}=\frac{\beta N-(\mu+\gamma)}{\beta+h(\mu+\gamma)} .
$$

Define

$$
V(I)=I-I^{*}-I^{*} \log \left(\frac{I}{I^{*}}\right) .
$$

Then $V$ is positive definite. By Itô formula, we yield

$$
\begin{aligned}
d V & =\left(1-\frac{I}{I^{*}}\right) d I+\frac{I^{*}}{2 I^{2}}(d I)^{2} \\
& =L V d t+\frac{\sigma(N-I)\left(I-I^{*}\right)}{1+h I} d W(t),
\end{aligned}
$$

where

$$
\begin{aligned}
L V & =\left(\frac{\beta(N-I)}{1+h I}-(\mu+\gamma)\right)\left(I-I^{*}\right)+\frac{I^{*} \sigma^{2}(N-I)^{2}}{2(1+h I)^{2}} \\
& =\left(\frac{\beta(N-I)}{1+h I}-\frac{\beta\left(N-I^{*}\right)}{1+h I^{*}}\right)\left(I-I^{*}\right)+\frac{I^{*} \sigma^{2}(N-I)^{2}}{2(1+h I)^{2}} \\
& =-\frac{\beta(1+h N)\left(I-I^{*}\right)^{2}}{(1+h I)\left(1+h I^{*}\right)}+\frac{I^{*} \sigma^{2}(N-I)^{2}}{2(1+h I)^{2}} \\
& \leq-\frac{\beta}{1+h I^{*}}\left(I-I^{*}\right)^{2}+\frac{1}{2} I^{*} \sigma^{2} N^{2} .
\end{aligned}
$$

Note that if $\sigma^{2}<\left(2 \beta / N^{2}\left(1+h I^{*}\right) I^{*}\right) \min \left\{\left(I^{*}\right)^{2},\left(N-I^{*}\right)^{2}\right\}$, then the neighborhood $U:-\left(\beta /\left(1+h I^{*}\right)\right)\left(I-I^{*}\right)^{2}+$ $(1 / 2) I^{*} \sigma^{2} N^{2}=0$ lies entirely in $(0, N)$. Hence, for $I \in$ $(0, N) \backslash U, L V \leq-k$ ( $k$ is a positive constant), which implies that condition (B.2) is satisfied; for more details, we refer to [27]. Therefore, system (5) has a stationary distribution.

Moreover,

$$
\begin{aligned}
0 & \leq E \int_{0}^{t} d V(I(s))=E \int_{0}^{t} L V(I(s)) d s \\
& \leq-E \int_{0}^{t} \frac{\beta}{1+h I^{*}}\left(I(s)-I^{*}\right)^{2} d s+\frac{1}{2} I^{*} \sigma^{2} N^{2} t .
\end{aligned}
$$

Then

$$
\lim _{t \rightarrow \infty} \frac{1}{t} E \int_{0}^{t} \frac{\beta}{1+h I^{*}}\left(I(s)-I^{*}\right)^{2} d s \leq \frac{1}{2} I^{*} \sigma^{2} N^{2} .
$$

Next, we will give the expressions for the mean and variance of the stationary distribution.

Theorem 10. If $R_{02}^{s}:=\beta N /((\mu+\gamma)(1+h N))-\sigma^{2} N^{2} /(2(\mu+$ $\gamma))>1$ and $\sigma^{2}<\left(2 \beta /\left(N^{2}\left(1+h I^{*}\right) I^{*}\right)\right) \min \left\{\left(I^{*}\right)^{2},\left(N-I^{*}\right)^{2}\right\}$. Let $m$ and $v$ denote the mean and variance of the stationary distribution of model (5). Then,

$$
\begin{gathered}
m=\left(\beta N-\mu-\gamma-\frac{1}{2} \sigma^{2} N^{2}\right) \\
\times\left(\left(\beta h+(\mu+\gamma) h^{2}+\frac{1}{2} \sigma^{2}\right) \frac{\beta N-\mu-\gamma}{\beta+(\mu+\gamma) h}\right. \\
\left.-\left(\beta(h N-1)-2 h(\mu+\gamma)+\sigma^{2} N\right)\right)^{-1} \\
v=\frac{\beta N-\mu-\gamma}{\beta+(\mu+\gamma) h} m-m^{2} .
\end{gathered}
$$

Proof. Multiply both sides of (5) by $1+h I(t)$; that is,

$$
\begin{aligned}
(1+ & h I(t)) d I(t) \\
= & (\beta(N-I(t)) I(t)-(\mu+\gamma) I(t)(1+h I(t))) d t \\
& +\sigma(N-I(t)) I(t) d W(t) .
\end{aligned}
$$




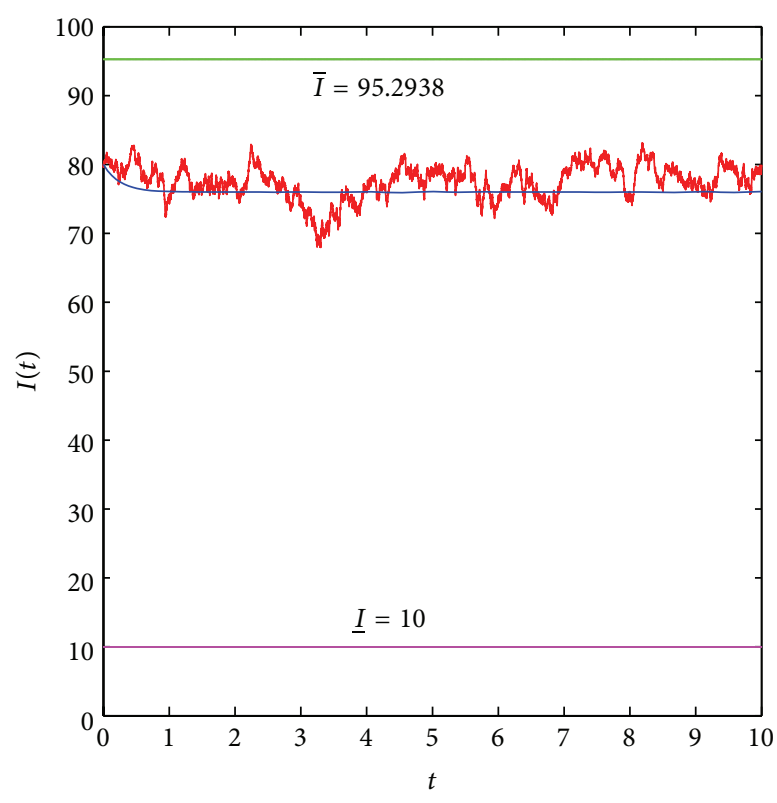

(a)

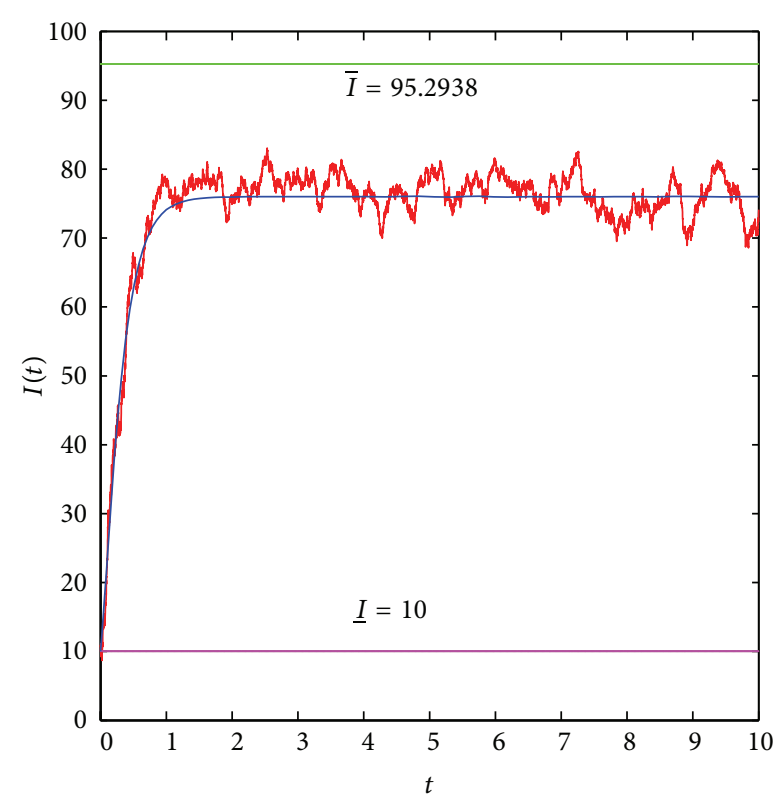

(b)

Figure 3: Computer simulations of $I(t)$ for model (5) (red line) and the deterministic model (1) (blue line), using the EM method with step size 0.001 and with initial values (a) $I(0)=80$ and (b) $I(0)=10 . \underline{I}=10$ and $\bar{I}=95.2938$.

Integrating (71) from 0 to $t$ and dividing by $t$ on both sides, then

$$
\begin{aligned}
\frac{1}{t} \int_{0}^{t}(1+h I(s)) d I(s) & \\
= & \frac{1}{t} \int_{0}^{t}(\beta(N-I(s)) I(s)-(\mu+\gamma) I(s)(1+h I(s))) d s \\
& +\frac{1}{t} \int_{0}^{t} \sigma(N-I(s)) I(s) d W(s) .
\end{aligned}
$$

Since

$$
\frac{I(t)-I_{0}}{t} \leq \frac{1}{t} \int_{0}^{t}(1+h I(s)) d I(s) \leq(1+h N) \frac{I(t)-I_{0}}{t}
$$

letting $t \rightarrow \infty$, we have

$$
\lim _{t \rightarrow \infty} \frac{1}{t} \int_{0}^{t}(1+h I(s)) d I(s)=0 \text { a.s. }
$$

Then, applying the ergodic property of the stationary distribution and strong law of large numbers, we get

$$
(\beta N-\mu-\gamma) m-(\beta+(\mu+\gamma) h)\left\langle I(t)^{2}\right\rangle=0 .
$$

Here, $\left\langle I(t)^{2}\right\rangle$ denotes the second moment of the stationary distribution [14].

In a similar way, multiplying both sides of (19) by $(1+$ $h I(t))^{2}$, we obtain that

$$
\begin{aligned}
(1+h I(t))^{2} d \log (I(t))= & (1+h I(t))^{2} F(I(t)) d t \\
& +\sigma(N-I(t))(1+h I(t)) d W(t) .
\end{aligned}
$$

Then, integrating (76) from 0 to $t$ and dividing by $t$ on both sides,

$$
\begin{aligned}
& \frac{1}{t} \int_{0}^{t}(1+h I(s))^{2} d \log (I(s)) \\
& =\frac{1}{t} \int_{0}^{t}(1+h I(s))^{2} F(I(s)) d s \\
& \quad+\frac{1}{t} \int_{0}^{t} \sigma(N-I(s))(1+h I(s)) d W(s) .
\end{aligned}
$$

Since

$$
\begin{aligned}
\frac{\log I(t)-\log I_{0}}{t} & \leq \frac{1}{t} \int_{0}^{t}(1+h I(s))^{2} d \log (I(s)) \\
& \leq(1+h N)^{2} \frac{\log I(t)-\log I_{0}}{t},
\end{aligned}
$$

then

$$
\lim _{t \rightarrow \infty} \frac{1}{t} \int_{0}^{t}(1+h I(s))^{2} d \log (I(s))=0 \quad \text { a.s. }
$$




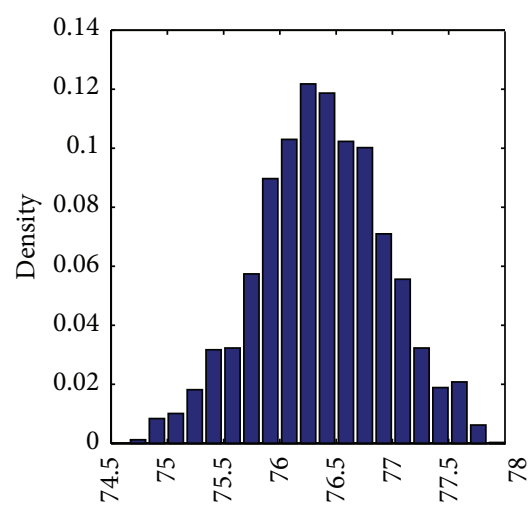

$I(t): \sigma=0.005$

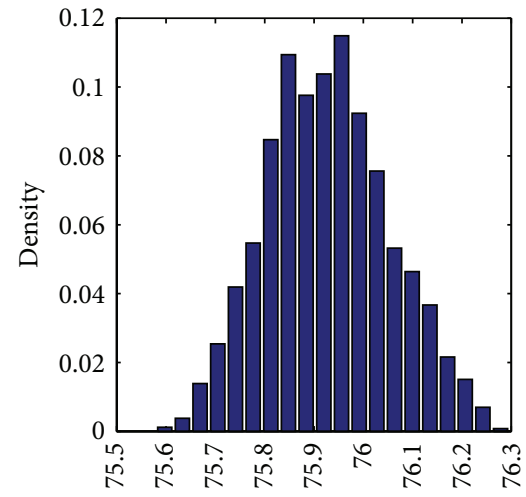

$I(t): \sigma=0.001$

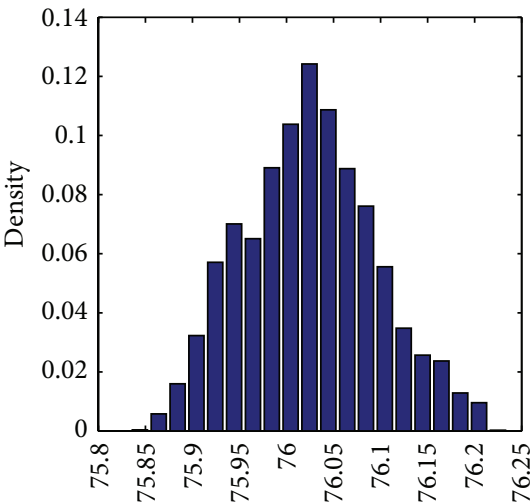

$I(t): \sigma=0.0005$

Figure 4: Histograms of the solution $I(t)$ for model (5) for $I(0)=10$ and differing values of $\sigma=0.005, \sigma=0.001$, and $\sigma=0.0005$.
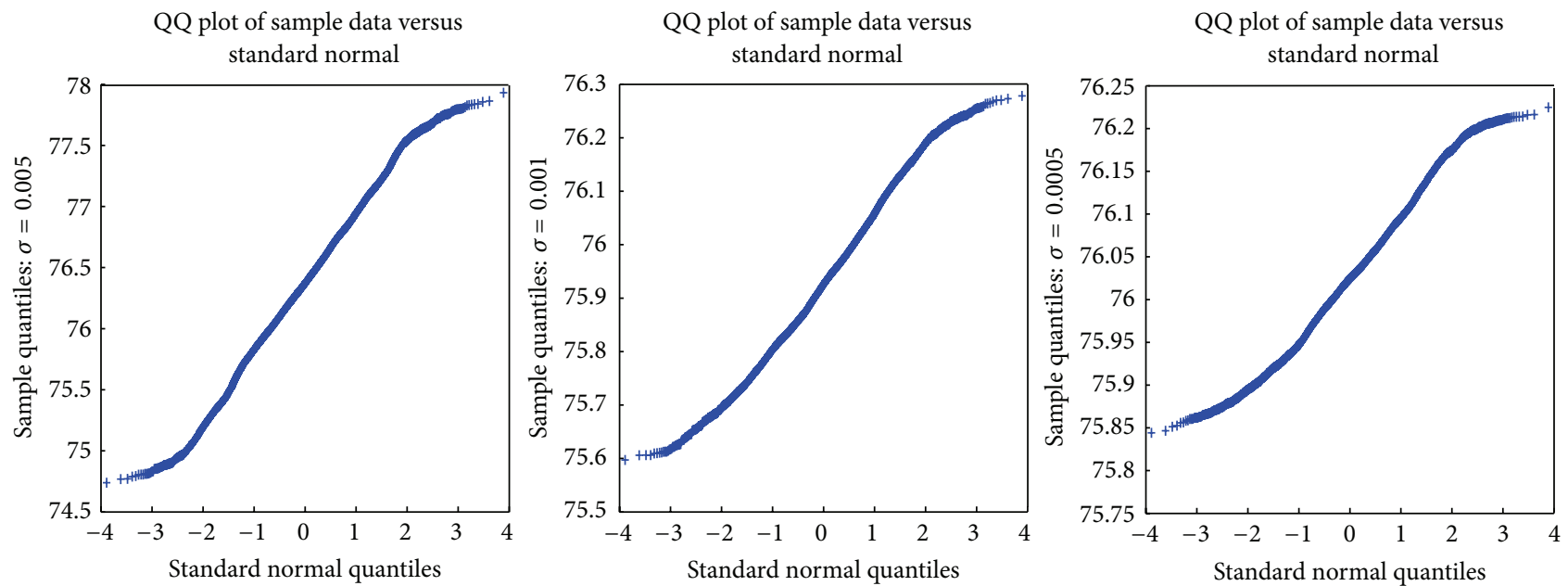

Figure 5: Normal quantile-quantile plots of the solution $I(t)$ for model (5) for $I(0)=10$ and differing values of $\sigma=0.005, \sigma=0.001$, and $\sigma=0.0005$.

Namely,

$$
\begin{aligned}
\lim _{t \rightarrow \infty} & \frac{1}{t} \int_{0}^{t}(1+h I(s))^{2} F(I(s)) d s \\
& +\lim _{t \rightarrow \infty} \frac{1}{t} \int_{0}^{t} \sigma(N-I(s))(1+h I(s)) d W(s)=0 \quad \text { a.s. }
\end{aligned}
$$

Therefore,

$$
\begin{gathered}
\beta N-\mu-\gamma-\frac{1}{2} \sigma^{2} N^{2}+\left(\beta(h N-1)-2 h(\mu+\gamma)+\sigma^{2} N\right) m \\
-\left(\beta h+(\mu+\gamma) h^{2}+\frac{1}{2} \sigma^{2}\right)\left\langle I(t)^{2}\right\rangle=0 .
\end{gathered}
$$

From (75) and (81), we can get

$$
\begin{aligned}
m=\left(\beta N-\mu-\gamma-\frac{1}{2} \sigma^{2} N^{2}\right) & \\
\times & \left(\left(\beta h+(\mu+\gamma) h^{2}+\frac{1}{2} \sigma^{2}\right) \frac{\beta N-\mu-\gamma}{\beta+(\mu+\gamma) h}\right. \\
& \left.\quad-\left(\beta(h N-1)-2 h(\mu+\gamma)+\sigma^{2} N\right)\right)^{-1},
\end{aligned}
$$

$$
v=\frac{\beta N-\mu-\gamma}{\beta+(\mu+\gamma) h} m-m^{2} .
$$

Example 11. We keep the parameters the same as Example 8 but reduce $\sigma$ to $0.005,0.001$, and 0.0005 . Note that $R_{02}^{s}$ is 


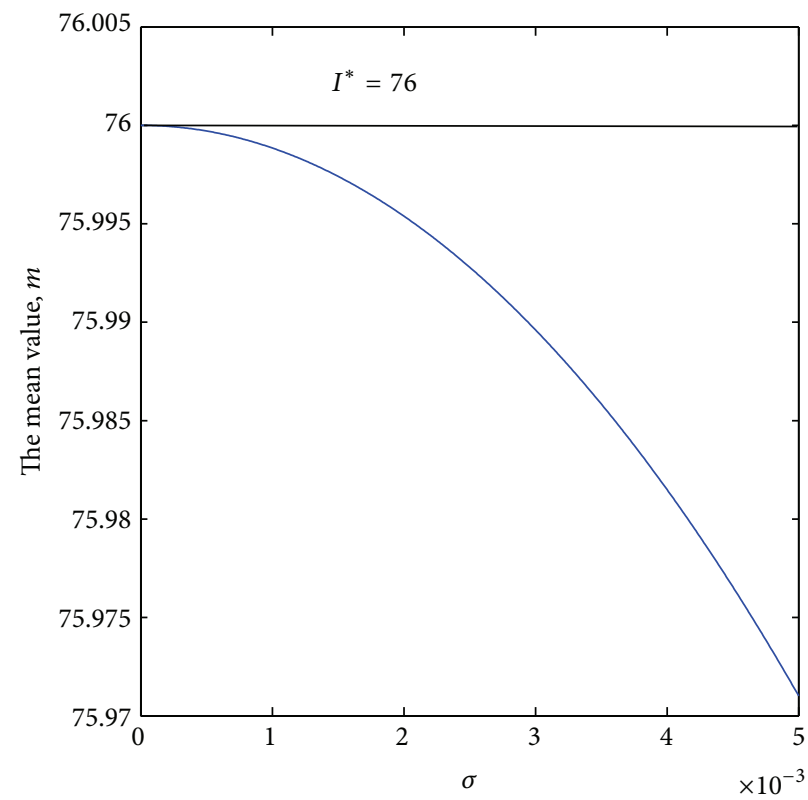

(a)

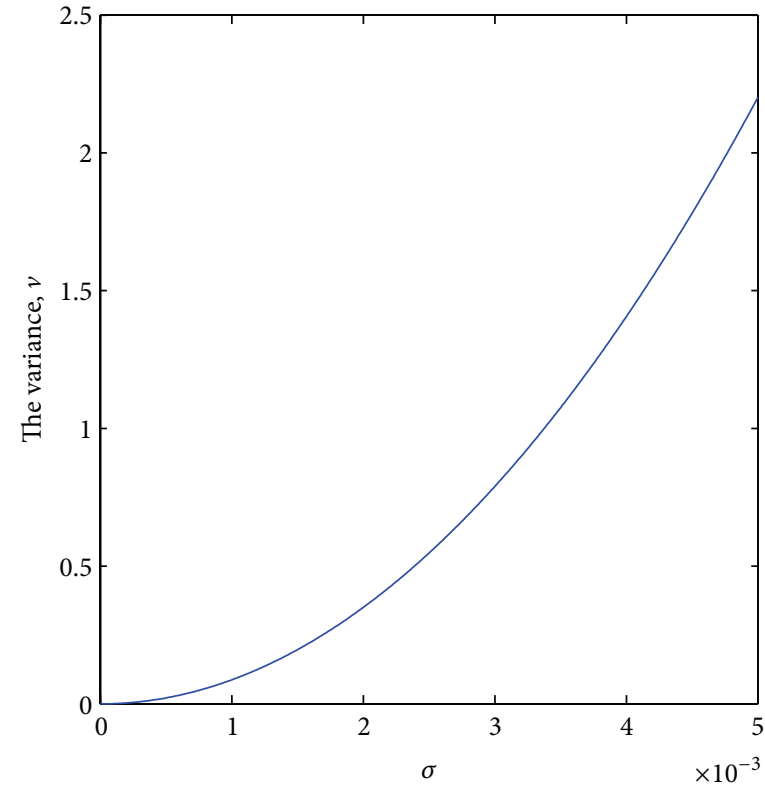

(b)

Figure 6: Plot the mean $m$ and variance $v$ against $\sigma$. The endemic equilibrium $I^{*}$ of the deterministic model (1) is 76.

3.2083, 3.3283, and 3.3321, respectively, and all of them are bigger than 1. Furthermore,

$$
\begin{gathered}
I^{*}=\frac{\beta N-(\mu+\gamma)}{\beta+h(\mu+\gamma)}=76, \\
\frac{2 \beta}{N^{2}\left(1+h I^{*}\right) I^{*}} \min \left\{\left(I^{*}\right)^{2},\left(N-I^{*}\right)^{2}\right\}=0.000063 .
\end{gathered}
$$

The values of $\sigma$ are satisfied for the conditions of Theorem 10 . The histograms of $I(t)$ are shown in Figure 4, respectively, for different values of $\sigma=0.005, \sigma=0.001$, and $\sigma=$ 0.0005 . The simulations were run for 100,000 iterations with step size 0.001 , and the first 90,000 iterations were discarded to allow $I(t)$ to reach its recurrent level. The distribution is slightly negative skewed for $\sigma=0.005$ and positive skewed for $\sigma=0.001$ and $\sigma=0.0005$. Additionally, the corresponding sample skewness coefficients are $-0.00067,0.0016$, and 0.0017 , separately. These simulation results illustrate that the distribution of $I(t)$ has reached the stationary distribution. When $\sigma=0.005$, from (70), the mean and variance of the stationary distribution are 75.9710 and 2.2016, respectively, while the sample mean and variance are 76.3754 and 0.3209 . For $\sigma=$ 0.001 , the mean and variance of the stationary distribution are 75.9988 and 0.0876 , compared to the sample mean and variance which are 75.9285 and 0.0157 , separately. When $\sigma$ reduces to 0.0005 , the mean and variance of the stationary distribution are 75.9997 and 0.0219 and the corresponding sample mean and variance are 76.0248 and 0.0050 . As $\sigma$ decreases, $I(t)$ becomes more symmetric about $I^{*}=76$ and the perturbations become much weaker. What is more, the normal quantile-quantile plots in Figure 5 suggest that these data are not far from being normally distributed.
From (70), we know that when $\sigma=0$, the mean value $m$ equals the value of the endemic equilibrium $I^{*}$ and the variance $v$ is zero. Furthermore, if $\sigma$ increases, the mean value $m$ decreases and the variance $v$ increases as shown in Figure 6. Obviously, the greater the intensity of the noise is, the larger the variance is, that is, the stronger the fluctuations of the distribution are. And this is in accordance with the actual case.

\section{Conclusion}

In this paper, we established a stochastic SIS epidemic model with saturated incidence to investigate the effect of environment noise. After proving the existence and uniqueness of the positive solution, we considered two kinds of stabilities: almost sure exponential stability and $p$ th moment exponential stability. Our deduction shows that the $p$ th moment exponential stability implies the almost sure exponential stability. As for the extinction of the disease, our results indicate that when the noise $\sigma^{2}$ is larger than $\beta(1+h N)^{2} / N \vee$ $\beta^{2}(1+h N)^{2} / 2(\mu+\gamma)$, the disease will die out a.s. If the noise is small, the disease will also die out a.s. when $R_{01}^{s}<$ 1 , instead, the disease will be persistent in the mean when $R_{02}^{s}>1$. Our investigation shows that the conditions for the disease to become extinct are much weaker than those in the corresponding deterministic model; that is, even if the disease dies out for the stochastic model, it may still persist for the corresponding deterministic model. Additionally, the half saturation constant influences the threshold value $R_{01}^{s}$, which is not obtained in the deterministic model. Increasing the saturation effect by changing the behavior of the susceptible individuals and taking effective control measures of the government can reduce $R_{01}^{s}$ and thus can reduce the spread 
of the disease. Finally, we proved the existence of a stationary distribution and derived the expressions for the mean and variance when the noise is small and $R_{02}^{s}>1$. Our theoretical results were further verified by computer simulations.

\section{Appendix}

First, we give some theory in stochastic differential equations.

Let $\left(\Omega, \mathscr{F},\left\{\mathscr{F}_{t}\right\}_{t \geq 0}, P\right)$ be a complete probability space with $\left\{\mathscr{F}_{t}\right\}_{t \geq 0}$ satisfying the usual conditions (i.e., it is increasing and right continuous while $\left\{\mathscr{F}_{0}\right\}$ contains all $P$-null sets). We use $a \vee b$ to denote $\max (a, b), a \wedge b$ to denote $\min (a, b)$, and a.s. to denote almost surely.

Definition A.1 (see [22]). Consider the $d$-dimensional stochastic differential equation

$$
d x(t)=f(x(t), t) d t+g(x(t), t) d B(t), \quad \text { for } t \geq t_{0},
$$

with initial value $x\left(t_{0}\right)=x_{0} \in R^{d}$ and $B(t)$ being the $m$-dimensional standard Brownian motion. The solution is represented as $x\left(t ; t_{0}, x_{0}\right)$. Assume that, for any $t \geq t_{0}$, there is

$$
f(0, t)=0, \quad g(0, t)=0 .
$$

So (A.1) has the solution $x(t) \equiv 0$. This solution is called the trivial solution.

Definition A.2 (see [22]). The trivial solution of (A.1) is said to be almost surely exponentially stable if

$$
\limsup _{t \rightarrow \infty} \frac{1}{t} \log \left|x\left(t ; t_{0}, x_{0}\right)\right|<0 \quad \text { a.s., }
$$

for all $x_{0} \in R^{d}$.

Definition A.3 (see [22]). The trivial solution of (A.1) is said to be $p$ th moment exponentially stable if there is a pair of positive constants $\lambda$ and $C$ such that

$$
E\left|x\left(t ; t_{0}, x_{0}\right)\right|^{p} \leq C\left|x_{0}\right|^{p} e^{-\lambda\left(t-t_{0}\right)} \quad \text { on } t \geq t_{0},
$$

for all $x_{0} \in R^{d}$. When $p=2$, it is said to be exponentially stable in mean square.

Lemma A.4 (see [22]). Assume that there is a positive constant $K$ such that

$$
x^{T} f(x, t) \vee|g(x, t)|^{2} \leq K|x|^{2} \quad \forall(x, t) \in R^{d} \times\left[t_{0}, \infty\right) .
$$

Then, the pth moment exponential stability of the trivial solution of (A.1) implies the almost sure exponential stability. [26].

Next, we give some theory about stationary distributions

Let $X(t)$ be a homogeneous Markov process in $E_{l}\left(E_{l}\right.$ denotes Euclidean $l$-space) described by

$$
d X(t)=f(X) d t+\sum_{s=1}^{m} g_{s}(X) d B_{s}(t)
$$

The diffusion matrix is $A(x)=\left(a_{i j}(x)\right), a_{i j}(x)=\sum_{s=1}^{m} g_{s}^{i}(x)$ $g_{s}^{j}(x)$.

Assumption B. There exists a bounded domain $U \subset E_{l}$ with regular boundary $\Gamma$, having the following properties.

(B.1) In the domain $U$ and some neighborhood thereof, the smallest eigenvalue of the diffusion matrix $A(x)=$ $\left(a_{i j}(x)\right)$ is bounded away from zero.

(B.2) If $x \in E_{l} \backslash U$, the mean time $\tau$ at which a path issuing from $x$ reaches the set $U$ is finite and $\sup _{x \in K} E_{x} \tau<\infty$ for every compact subset $K \subset E_{l}$.

Lemma A.5 (see [26]). If (B.1) and (B.2) hold, then the Markov process $X(t)$ has a stationary distribution.

\section{Conflict of Interests}

The authors declare that there is no conflict of interests regarding the publication of this paper.

\section{Acknowledgment}

The work is financially supported by the National Natural Science Foundation of China (nos. 11072182 and 11372233).

\section{References}

[1] S. Busenberg and P. van den Driessche, "Analysis of a disease transmission model in a population with varying size," Journal of Mathematical Biology, vol. 28, no. 3, pp. 257-270, 1990.

[2] M. Y. Li and J. S. Muldowney, "Global stability for the SEIR model in epidemiology," Mathematical Biosciences, vol. 125, no. 2, pp. 155-164, 1995.

[3] M. Fan, M. Y. Li, and K. Wang, "Global stability of an SEIS epidemic model with recruitment and a varying total population size," Mathematical Biosciences, vol. 170, no. 2, pp. 199-208, 2001.

[4] J. Q. Li, J. Zhang, and Z. E. Ma, "Global analysis of some epidemic models with general contact rate and constant immigration," Applied Mathematics and Mechanics, vol. 25, no. 4, pp. 396-404, 2004.

[5] X.-Q. Zhao and X. Zou, "Threshold dynamics in a delayed SIS epidemic model," Journal of Mathematical Analysis and Applications, vol. 257, no. 2, pp. 282-291, 2001.

[6] M. Safan and F. A. Rihan, "Mathematical analysis of an SIS model with imperfect vaccination and backward bifurcation," Mathematics and Computers in Simulation, vol. 96, pp. 195-206, 2014.

[7] S. Tang, Y. Xiao, L. Yuan, R. A. Cheke, and J. Wu, "Campus quarantine (Fengxiao) for curbing emergent infectious diseases: lessons from mitigating $\mathrm{A} / \mathrm{H} 1 \mathrm{~N} 1$ in Xian, China," Journal of Theoretical Biology, vol. 295, pp. 47-58, 2012.

[8] K. Dietz, "Overall population patterns in the transmission cycle of infectious disease agents," in Population Biology of Infectious Disease, Springer, 1982.

[9] X. Zhang and X. Liu, "Backward bifurcation of an epidemic model with saturated treatment function," Journal of Mathematical Analysis and Applications, vol. 348, no. 1, pp. 433-443, 2008. 
[10] Y. Lin, D. Jiang, and S. Wang, "Stationary distribution of a stochastic SIS epidemic model with vaccination," Physica A, vol. 394, pp. 187-197, 2014.

[11] Q. Yang and X. Mao, "Extinction and recurrence of multigroup SEIR epidemic models with stochastic perturbations," Nonlinear Analysis: Real World Applications, vol. 14, no. 3, pp. 1434-1456, 2013.

[12] Y. Zhao and D. Jiang, "Dynamics of stochastically perturbed SIS epidemic model with vaccination," Abstract and Applied Analysis, vol. 2013, Article ID 517439, 12 pages, 2013.

[13] Y. Zhao and D. Jiang, "The threshold of a stochastic SIRS epidemic model with saturated incidence," Applied Mathematics Letters, 2013.

[14] A. Gray, D. Greenhalgh, L. Hu, X. Mao, and J. Pan, "A stochastic differential equation SIS epidemic model," SIAM Journal on Applied Mathematics, vol. 71, no. 3, pp. 876-902, 2011.

[15] S. Tornatore, S. Buccellato, and P. Vetro, "Stability of a stochastic SIR system," Physica A, vol. 354, pp. 111-126, 2005.

[16] Q. Lu, "Stability of SIRS system with random perturbations," Physica A, vol. 388, no. 18, pp. 3677-3686, 2009.

[17] Q. Yang, D. Jiang, N. Shi, and C. Ji, "The ergodicity and extinction of stochastically perturbed SIR and SEIR epidemic models with saturated incidence," Journal of Mathematical Analysis and Applications, vol. 388, no. 1, pp. 248-271, 2012.

[18] C. Ji, D. Jiang, Q. Yang, and N. Shi, "Dynamics of a multigroup SIR epidemic model with stochastic perturbation," Automatica, vol. 48, no. 1, pp. 121-131, 2012.

[19] C. Ji, D. Jiang, and N. Shi, "Multigroup SIR epidemic model with stochastic perturbation," Physica A, vol. 390, pp. 1747-1762, 2011.

[20] Y. Zhao, D. Jiang, and D. O'Regan, "The extinction and persistence of the stochastic SIS epidemic model with vaccination," Physica A, vol. 392, no. 20, pp. 4916-4927, 2013.

[21] N. Dalal, D. Greenhalgh, and X. Mao, "A stochastic model of AIDS and condom use," Journal of Mathematical Analysis and Applications, vol. 325, no. 1, pp. 36-53, 2007.

[22] X. Mao, Stochastic Differential Equations and Their Applications, Horwood, Chinester, UK, 1997.

[23] D. J. Higham, "An algorithmic introduction to numerical simulation of stochastic differential equations," SIAM Review, vol. 43, no. 3, pp. 525-546, 2001.

[24] Y. Ding, M. Xu, and L. Hu, "Asymptotic behavior and stability of a stochastic model for AIDS transmission," Applied Mathematics and Computation, vol. 204, no. 1, pp. 99-108, 2008.

[25] L. Chen and J. Chen, Nonlinear Biological Dynamical System, Science, Beijing, China, 1993.

[26] R. Z. Hasminskii, Stochastic Stability of Differential Equations, Sijthoff \& Noordhoff, Alphen aan den Rijn, The Netherlands, 1980.

[27] C. Zhu and G. Yin, "Asymptotic properties of hybrid diffusion systems," SIAM Journal on Control and Optimization, vol. 46, no. 4, pp. 1155-1179, 2007. 


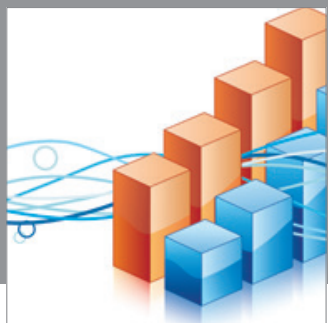

Advances in

Operations Research

mansans

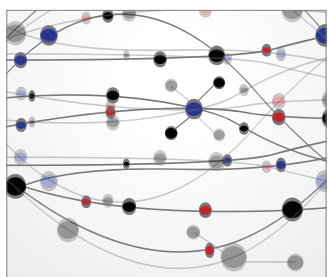

The Scientific World Journal
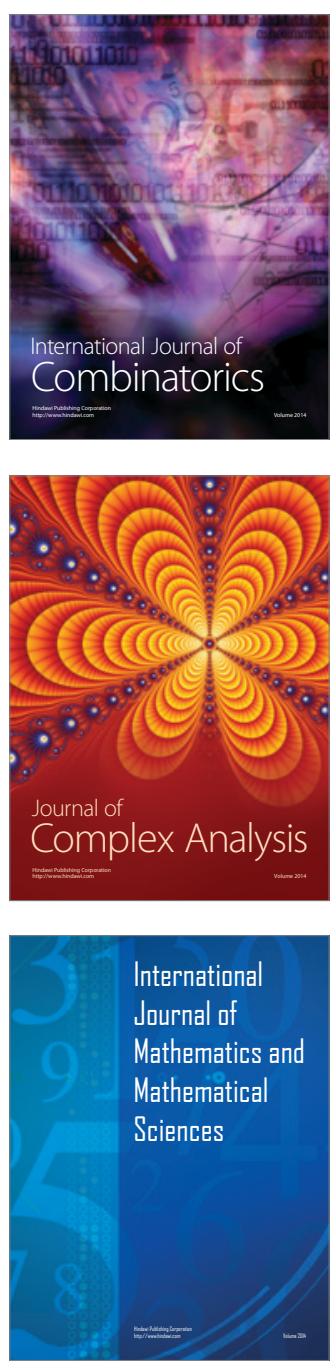
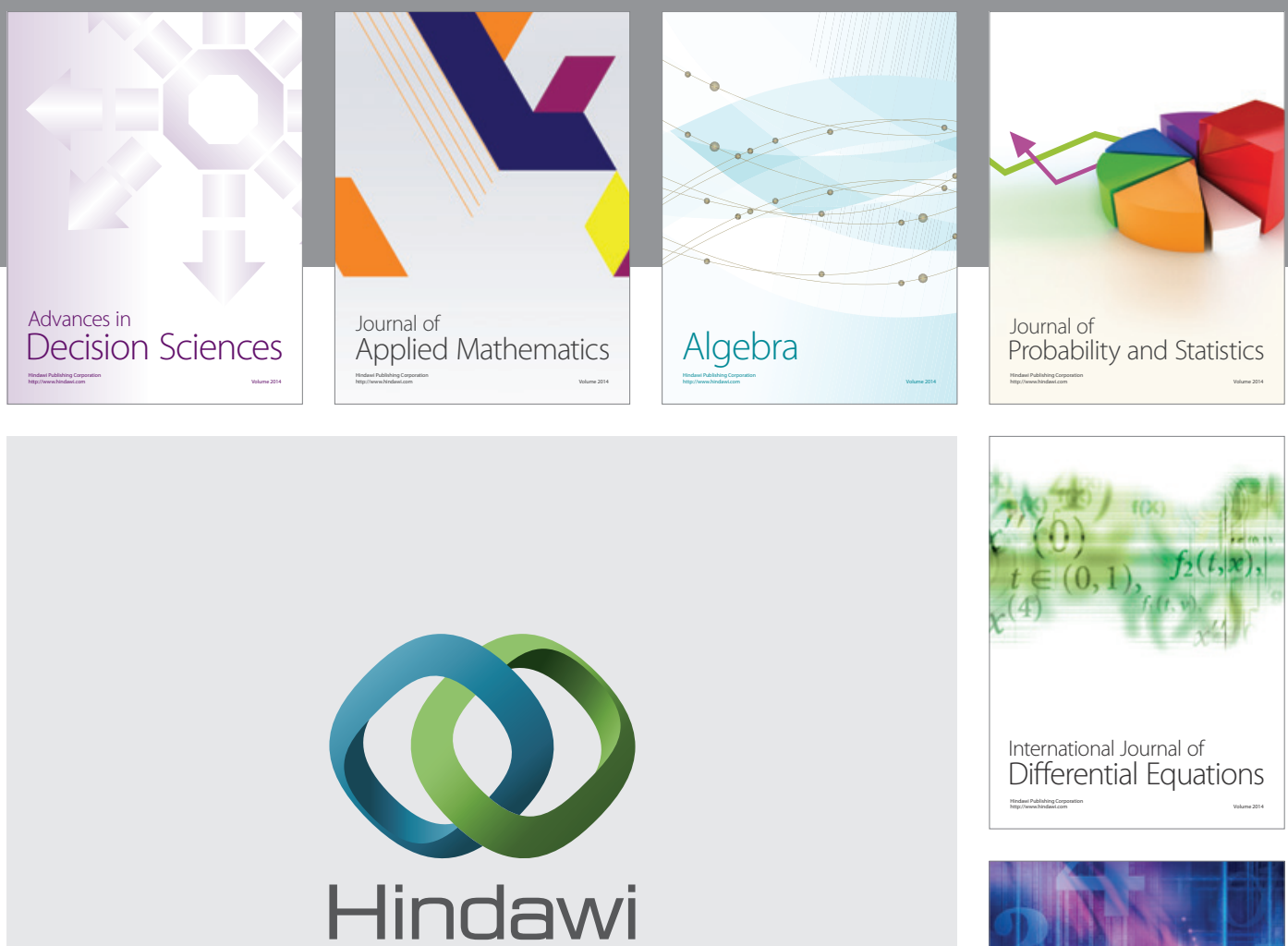

Submit your manuscripts at http://www.hindawi.com
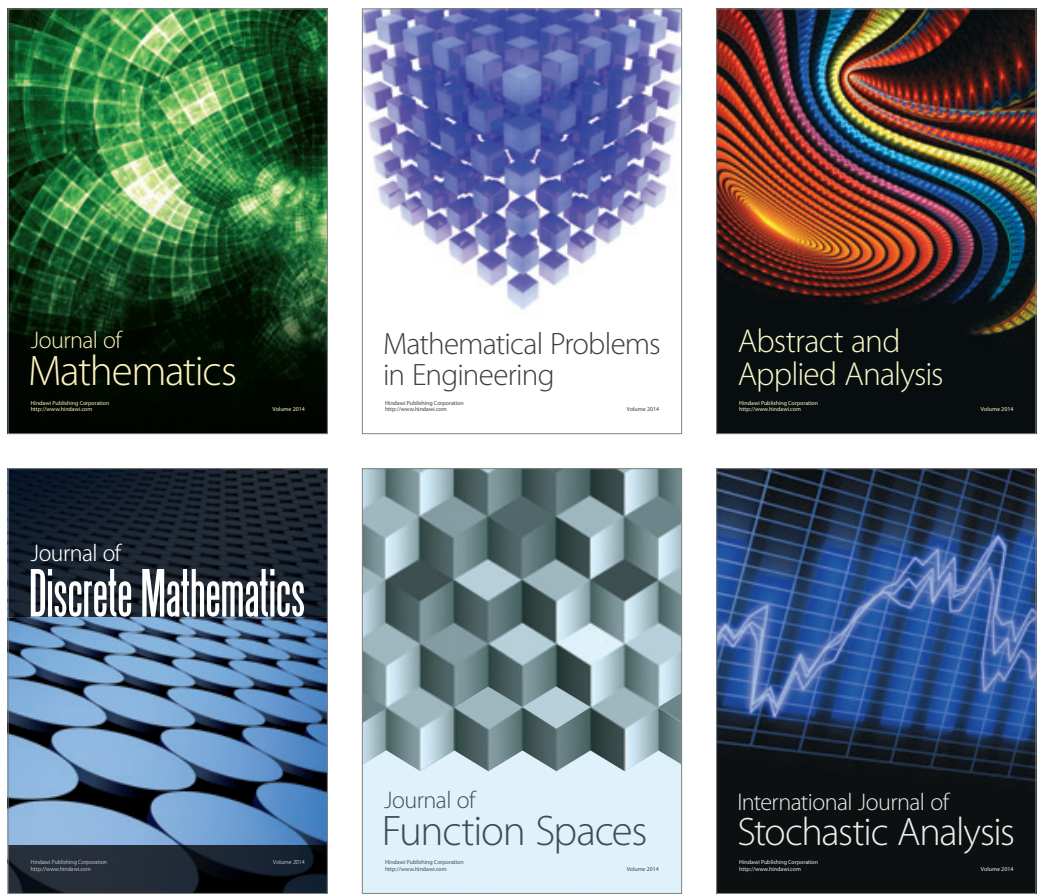

Journal of

Function Spaces

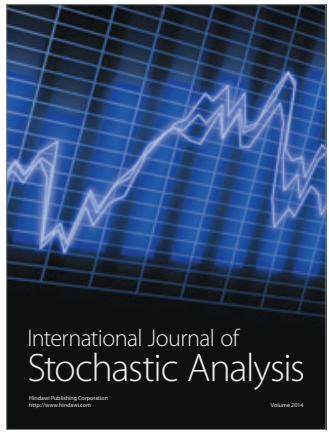

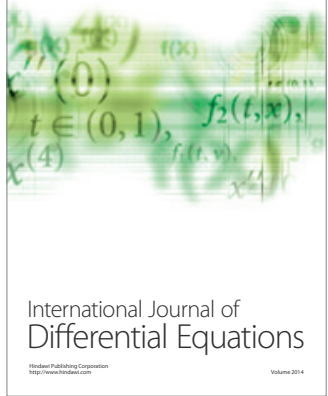
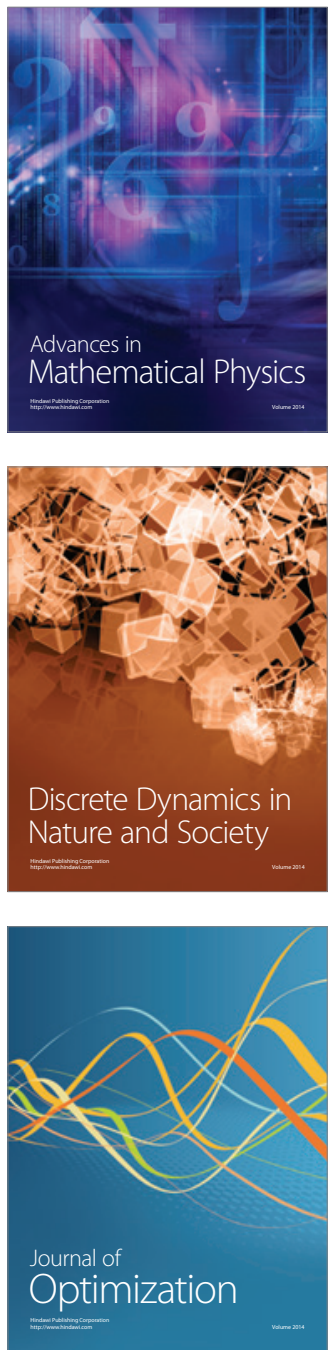